\title{
Robust Multimodality Registration for Brain Mapping
}

\section{Laurent Itti, ${ }^{1,2 *}$ Linda Chang, ${ }^{1}$ Jean-François Mangin, ${ }^{2,3}$ Jacques Darcourt ${ }^{4}$ and Thomas Ernst ${ }^{1,5}$}

\author{
${ }^{1}$ Department of Neurology, Harbor-UCLA Medical Center, Torrance, California 90509 \\ ${ }^{2}$ Département Images, Ecole Nationale Supérieure des Télécommunications, Paris, France 75014 \\ ${ }^{3}$ Commissariat á l'Energie Atomique, Service Hospitalier Fréderic Joliot, Orsay, France 91406 \\ ${ }^{4}$ Service de Médecine Nucléaire, Centre Antoine Lacassagne, Nice, France 06189 \\ ${ }^{5}$ Department of Radiolody, Harbor-UCLA Medical Center, Torrance, California 90509
}

\begin{abstract}
We present a robust intrasubject registration method for the synergistic use of multiple neuroimaging modalities, with applications to magnetic resonance imaging (MRI), functional MRI, perfusion MRI, MR spectroscopy, and single-photon emission computed tomography (SPECT). This method allows user-friendly processing of difficult examinations (low spatial resolution, advanced pathology, motion during acquisition, and large areas of focal activation). Registration of three-dimensional (3D) brain scans is initially estimated by first-order moment matching, followed by iterative anisotrophic chamfer matching of brain surfaces. Automatic brain surface extraction is performed in all imaging modalities. A new generalized distance definition and new specific methodologies allow registration of scans that cover only a limited range of brain surface. A new semiautomated supervision scheme allows fast and intuitive corrections of possible false automatic registration results. The accuracy of the MRI/SPECT anatomical-functional correspondence obtained was evaluated using simulations and two difficult clinical populations (tumors and degenerative brain disorders). The average discrimination capability of SPECT (12.4 mm in-plane resolution, $20 \mathrm{~mm}$ slice thickness) was found to be better than $5 \mathrm{~mm}$ after registration with MRI (5 mm slice thickness). Registration accuracy was always better than imaging resolution. Complete 3D MRI and SPECT registration time ranged between 6-11 min, in which surface matching represented 2-3 min. No registration failure occurred. In conclusion, the application of several new image processing techniques allowed efficient and robust registration. Hum. Brain Mapping 5:3-17, 1997. 1997 Wiley-Liss, Inc.
\end{abstract}

Key words: multimodality registration; neuroimaging; brain; chamfer matching; brain surface; image processing

\section{INTRODUCTION}

A variety of physical principles are applied in modern neuroimaging techniques such as magnetic

Contract Grant Sponsor: French Foundation for Alzheimer Research (Augustus Rose Fellowship); Contract Grant sponsor: NIH General Clinical Research Center; Contract Grant number: MO1 RR00425; Contract Grant sponsor: Clinical Associate Physician, Scientist Development Award for Clinicians; Contract Grant number: DA00280; Contract Grant sponsor: UCLA Alzheimer Disease Center; Contract Grant number: Ag-10123-03.

${ }^{*}$ Correspondence to: Laurent Itti, now at the Department of Computation and Neural Systems, California Institute of Technology, Mail Code 139-74, Pasadena, CA 91125.

Received for publication 26 February 1996; accepted 12 December 1996 resonance imaging (MRI), functional MRI (fMRI), perfusion MRI (pMRI), magnetic resonance spectroscopy (MRS), single photon emission computed tomography (SPECT), and positron emission tomography (PET). These different mechanisms make simple comparisons and synergistic use of the images for brain mapping difficult and often ambiguous. A major problem is the precise localization of physically corresponding regions in multiple data sets. The goal of multimodality registration is to fuse two or more sets of images in order to combine the information from each technique into a composite correlated data set. This requires a reliable physical correspondence, i.e., a registration transformation, to be established between the original

(C) 1997 Wiley-Liss, Inc. 
images. Such a correspondence is not known a priori, because considerable positioning freedom is allowed in imaging devices. Applications of mono- and multimodality registration have been reviewed in several papers [Levin et al., 1988; van den Elsen et al., 1993]. In particular, the understanding of the relationship between brain structure (MRI) and function (fMRI, pMRI, SPECT, PET, and MRS) is of major importance for differentiating diseases and for understanding how brain physiology maps onto brain anatomy. For example, the applicability of SPECT to focal activation studies has been demonstrated by Crosson et al. [1994] and Yoshizawa et al. [1995] and would greatly benefit from correlation with anatomy and other functional modalities. New techniques such as pMRI and MRS would also benefit from registration with other anatomical and functional imaging modalities.

We are particularly interested in registration of routine clinical examinations, because we believe that one of the most promising applications of anatomicalfunctional brain mapping is in the study of brain disorders. Therefore, our registration method was developed to allow processing of difficult data, such as low-resolution SPECT images (12-20 mm resolution) and relatively low-resolution MR acquisitions (5-8 $\mathrm{mm}$ slice thickness). Registration in the clinical setting also might encounter technical difficulties (due to motion during the acquisitions or variability of imaging sequences and agents) and a high variability of the populations studied (such as focal activation, advanced brain degeneration, surgical resections, and structural abnormalities).

Our method was consequently defined by the following eight guidelines: 1) Only intrasubject registration would be performed, with the assumption that the imaged brain was not subject to deformations [van den Elsen et al., 1993]. Consequently, global rigid transformations (three-dimensional (3D) rotation, 3D translation, and 3D uniform scaling) would be performed. 2) No external markers would be required [Ende et al., 1991; Fright and Linney, 1993; Loats, 1993; Grabowski et al., 1995], which would allow retrospective registration. 3) No constraint would exist concerning the orientation, resolution, slice thickness or spacing, and total number of slices in each study. 4) Registration of scans covering only part of the brain as well as full head examinations would be possible. This requirement might pose difficulties to some moments-based methods [Alpert et al., 1990; Moshfeghi and Rusinek, 1992; Arata and Dhawan, 1992] and to some surfacebased methods: e.g., a partial sagittal MRI of the left half of the head and a partial axial SPECT have different axes of inertia as well as different surfaces (but see Dhawan et al. [1995] for more developments on this point). 5) Unless necessary, internal (anatomical) landmarks would not be used to guide registration [Wang et al., 1994], because most such landmarks are difficult to precisely delineate in our low-resolution SPECT. 6) Methods based on comparisons of image intensities [Woods et al., 1993; Friston et al., 1995] did not seem very well-adapted to our problem, since little correlation may exist between some of our imaging modalities (see Results, concerning the registration of MRI to thallium SPECT, in which most of the brain except for tumors and choroid plexus appears as noise). 7) Interactive matching methods [Evans et al., 1991; Wang et al., 1994] were avoided if automatic methods could be devised. 8) The process needed to be robust enough to allow registration of virtually any set of images from a large number of modalities, while remaining highly or fully automatic.

Our method is an extension of the surface-based paradigm [Pelizzari et al., 1989; Holman et al., 1991; Besl and McKay, 1992; Jiang et al., 1992; Mangin et al., 1994; van Herk and Kooy, 1994, Turkington et al, 1995]. It has been designed to meet our objectives, with particular attention given to the problem of registering low-resolution, technically imperfect data sets.

\section{MATERIALS AND METHODS}

\section{Image acquisition and processing hardware}

MRI was performed on a clinical (non-echo planar) 1.5 Tesla General Electric SIGNA imaging system (General Electric Medical Systems, Milwaukee, WI) in axial, sagittal, or coronal orientations. MR sequences used for structural imaging were $T_{1}, T_{2}, T_{1}$ with contrast agent, 3D spoiled-grass, inversion recovery, and multiecho. Localized ${ }^{1} \mathrm{H}$ MR spectroscopy was performed using a point resolved spectroscopy (PRESS) sequence [Bottomley, 1987] (echo time (TE) $30 \mathrm{msec}$, relaxation time (TR) $3 \mathrm{sec}, 64$ averages). Absolute quantitation of the spectra was achieved as previously described [Ernst et al., 1993; Kreis et al., 1993].

Perfusion MRI (pMRI) was performed on a single 8 -mm slice using a gradient echo sequence (TE 18 $\mathrm{msec}$, TR $40 \mathrm{msec}$, flip angle $10^{\circ}, 2.5 \mathrm{sec}$ per scan). This slice was acquired sequentially 40 times, yielding a total scan time of $1 \mathrm{~min} 43 \mathrm{sec}$. Twenty $\mathrm{ml}$ of gadolinium contrast agent Prohance (Squibb, Princeton, NJ) was injected as an intravenous bolus. Perfusion maps were then computed using a numerical integration algorithm developed in our laboratory.

Cortical activation during visual or motor stimulation was observed using blood oxygenation leveldependent (BOLD) fMRI contrast. Five oblique slices 
through the primary visual or motor cortices were scanned using a spoiled gradient echo sequence (TE 50 msec, TR $60 \mathrm{msec}, 1$ excitation, 64 phase-encoding steps, flip angle $10^{\circ}$, field of view $48 \mathrm{~cm}$, $1.875 \times 1.875 \times 8 \mathrm{~mm}^{3}$ voxels). A T2-weighted structural localizer was used to position the five slices for fMRI. Visual stimulation was performed by flashing a pair of goggles with a frequency of $4 \mathrm{~Hz}$, and motor stimulation consisted of a simple finger-tapping task. The activation protocol comprised two baseline scans (five slices each), two scans during stimulation, and two more baseline and stimulated scans, yielding a total of eight images per slice. The images were processed using an IDL-based program (Research Systems, Boulder, CO) to compute a z-map.

SPECT studies were performed with a Shimadzu Headtome II dedicated brain unit (Shimadzu, Kyoto, Japan). It provides 11 nonuniformly-spaced slices (four bed shifts with three fixed ring detectors, one slice being redundant), with a spatial resolution of $12.4 \mathrm{~mm}$ in plane (full width at half maximum, FWHM) and 20 $\mathrm{mm}$ slice thickness. Spatial oversampling performed by the camera's reconstruction software yielded $3.3 \times$ $3.3 \times 20 \mathrm{~mm}^{3}$ voxels. A variety of agents was used: HMPAO (technetium-99m hexamethyl propyleneamine oxime; Ceretec, Amersham, Arlington Heights, IL) for relative cerebral blood flow [Chang et al., 1994; Masdeu et al., 1994]; ${ }^{133}$ Xe (Xenon-133;Dupont, Billerica, MA) for absolute blood flow measurements and calibration of HMPAO images [Kanno and Lassen, 1979]; and MIBI (technetium-99m methoxy-isobutyl-isonitrile; Cardiolite, Dupont-Merck, Billerica, MA) and ${ }^{201} \mathrm{Tl}$ (201-thallous chloride, Mallinckrodt, Maryland Heights, MD) for tumor metabolism studies [Chiu et al., 1990; Masdeu et al., 1994]. Standard SPECT images with parallelepipedic voxels $\left(6.25 \times 6.25 \times 6.25 \mathrm{~mm}^{3}\right)$, acquired on a Sophy Camera DSX (Sopha Medical, Columbia, MD), were also used.

The registration package was developed on a Sun SPARCStation LX (Sun Microsystems, Mountain View, CA), using the AVS graphical interface and objectoriented development tool (Advanced Visual Systems, Waltham, MA) and specifically written (language C) AVS modules and networks of modules.

\section{Automated brain surface segmentation}

All images were converted from their original 16-bit intensity range to 8-bit range, in order to halve memory requirements during processing. The marginal surface of the brain was used for registration, since it is one of the few large structures visible in all imaging modalities. Automatic extraction of this surface was achieved by an extended version of the method of Malandain [1992]. His method, as well as those of Mangin et al. [1994] and Alpert et al. [1996], use binary morphological erosion to separate the brain from surrounding tissues, followed by detection of the brain as the largest 3D connected component, and binary morphological dilation to recover original brain size. We extended this method by first providing an automatic interpretation of the intensity distribution histogram of the volume. This eliminates all objects unambiguously outside brain intensity range by applying an upper and lower threshold to binarize the volume. For example, with $\mathrm{T}_{1}$-weighted $\mathrm{MR}$ images, the lower threshold $\mathrm{T}_{\min }$ eliminates the background noise and skull, while the higher threshold $\mathrm{T}_{\max }$ eliminates most of the fat. The interpretation algorithm first applies median filtering and boxcar smoothing to the histogram. The local minimum towards lower intensities $\mathrm{m}$ is then detected (Fig. 1, top), and separates background noise (towards lower intensities) from signal (towards higher intensities). The maximum $\mathrm{M}$ of the histogram for intensities higher than $\mathrm{m}$ is then detected and usually corresponds to a white-matter intensity. The lower threshold $\mathrm{T}_{\min }$ is computed as a linear combination of $\mathrm{m}, \mathrm{M}$, and the point between $\mathrm{m}$ and $\mathrm{M}$ showing the most abrupt change in the histogram's slope. The upper threshold $\mathrm{T}_{\max }$ is determined by a linear combination between $\mathrm{M}$ and the point with intensities higher than $M$ showing the most abrupt change in slope. The parameters for these linear combinations were determined empirically for three broad classes of MR sequences $\left(T_{1} /\right.$ proton-density/first echo, $T_{2} /$ second echo, and inversion-recovery). The resulting volume contained a binarized representation of the brain, and of objects with similar intensities (e.g., parts of the skin, muscles, and nerves). At this stage, the separation between brain and surrounding tissues was imperfect in several locations (Fig. 1).

An isotropic 3D binary morphological erosion [Schmitt and Mattioli, 1994] of the thresholded volume was consequently applied to separate the brain from adjacent objects. Contracy to 2D methods, 3D erosion accentuates brain contours not only within slices, but also towards adjacent slices, which is important when only partial brain contours are present in some slices after the initial thresholding. Fast 3D erosion is obtained by thresholding a chamfer distance map [Wacken, 1994]. This fast implementation is equivalent to using the conventional implementation with a structuring element that is a solid sphere for the chamfer distance used, with radius equal to the threshold applied to the distance map. Although this sphere is defined by all points equidistant from its center, it 

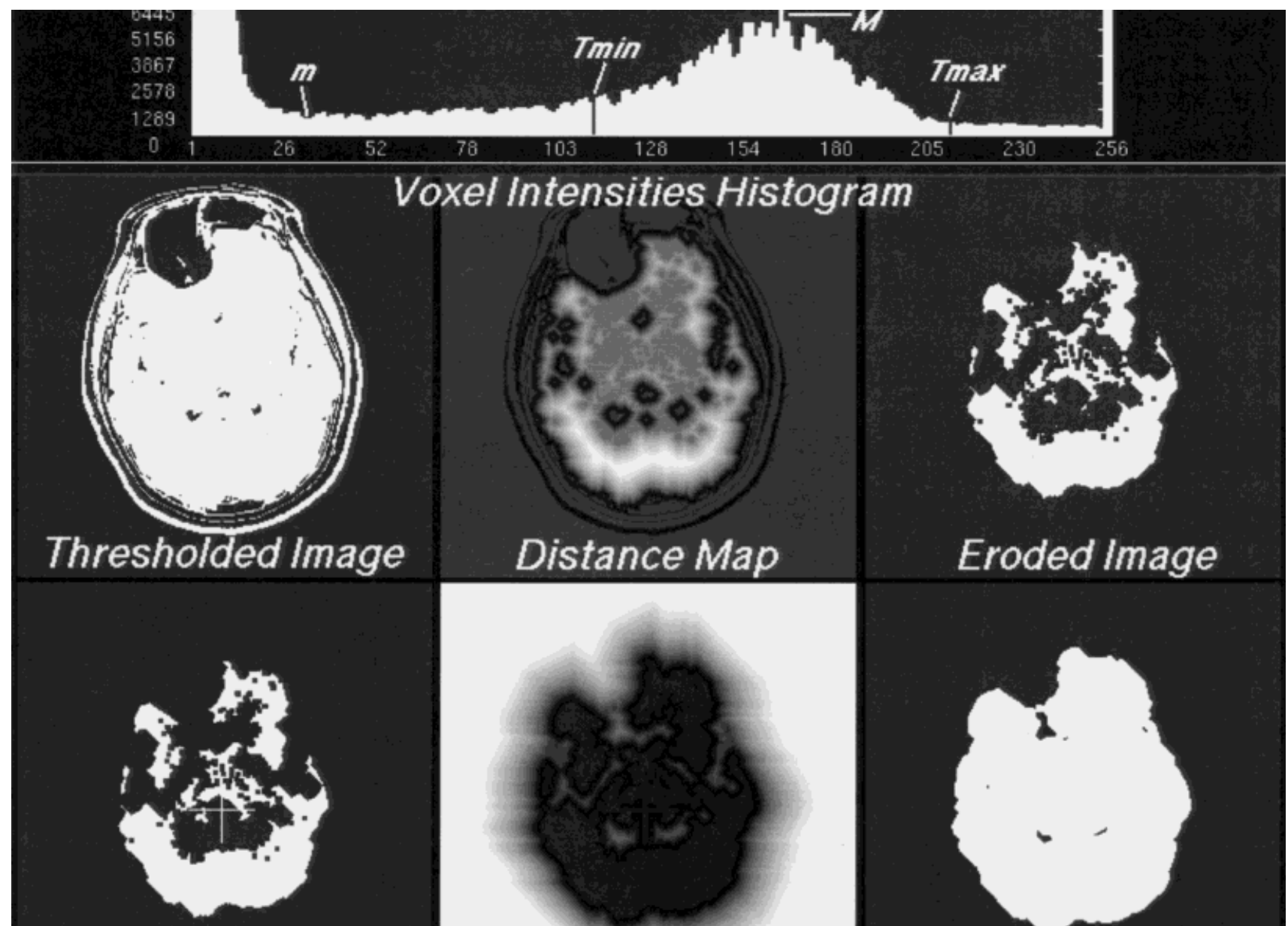

\section{Connected Brain}
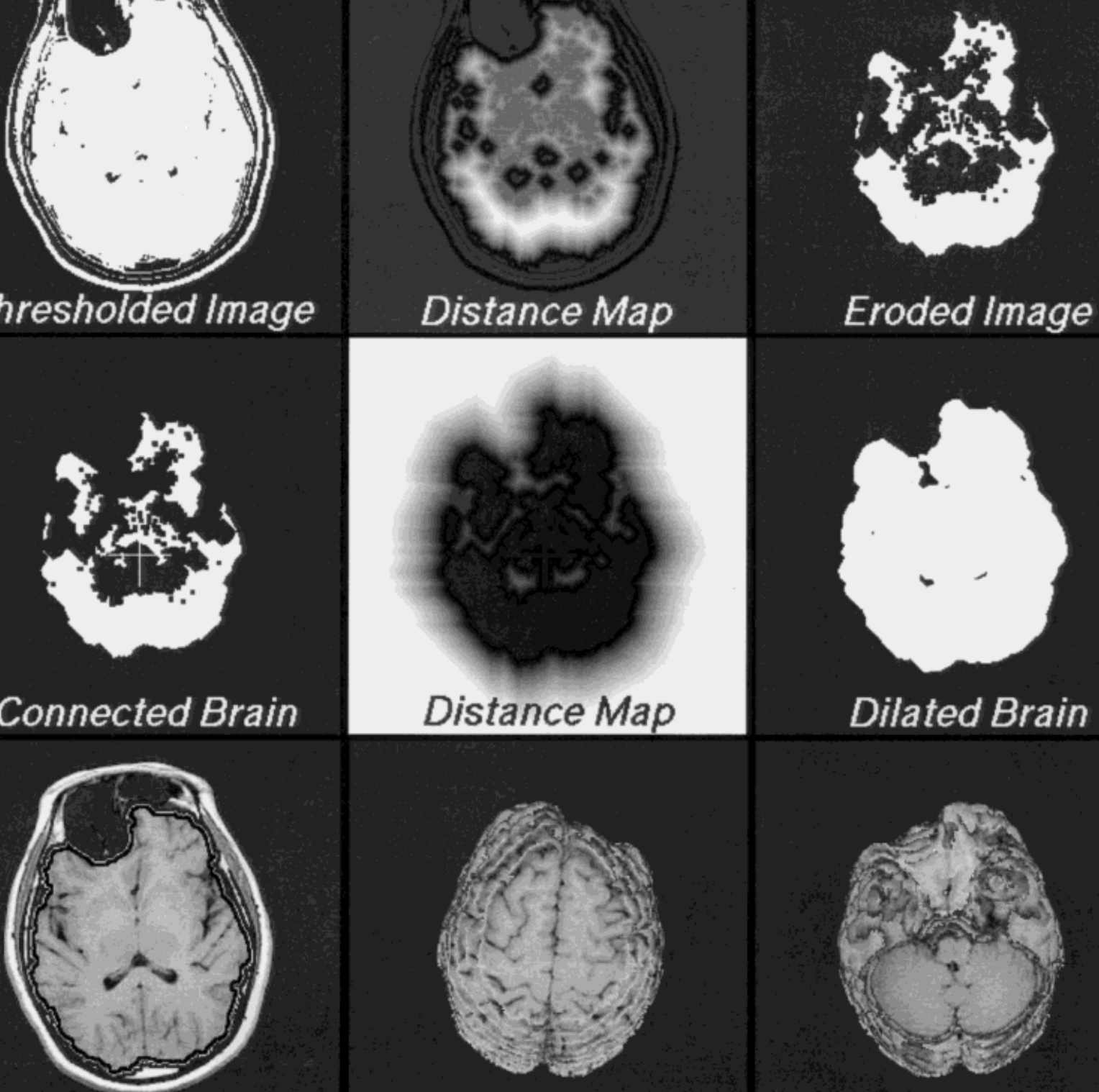

Original + Contours

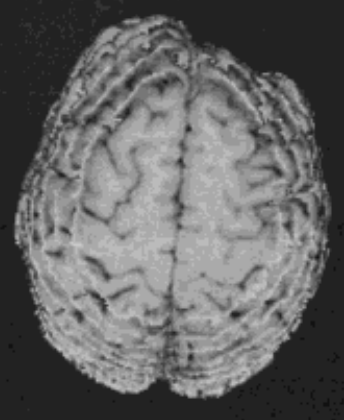

$3 D$ Top View

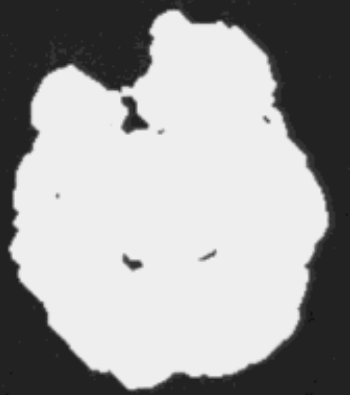

Dilated Brain

Figure 1.

Visual outline (from left to right, top to bottom) of our automatic brain segmentation method, applied without manual intervention, to the MRI of a patient with a large right frontal defect (enlarged sinuses). The MRI volume had $0.9375 \times 0.9375 \times 5 \mathrm{~mm}^{3}$ voxels and 26 slices (first echo of TR $=2,500 \mathrm{msec}, \mathrm{TE}=30$ and 100 msec, 2 excitations, 192 views; second echo was not used). A utomatic double thresholding (top left) based on the gray-level histogram (top) allowed most brain contours to be seen, although they were mixed with other contours and only partly defined. Morphological isotropic erosion (efficiently computed by thresholding an anisotropic distance map) accentuated these contours. 3D flooding of the brain then isolated it from remaining neighboring masses, and morphological dilation (computed by thresholding another distance map) recovered its original size. In regions where brain contours were present in the initial thresholded image (top left), they were used to refine the dilated brain shape. 

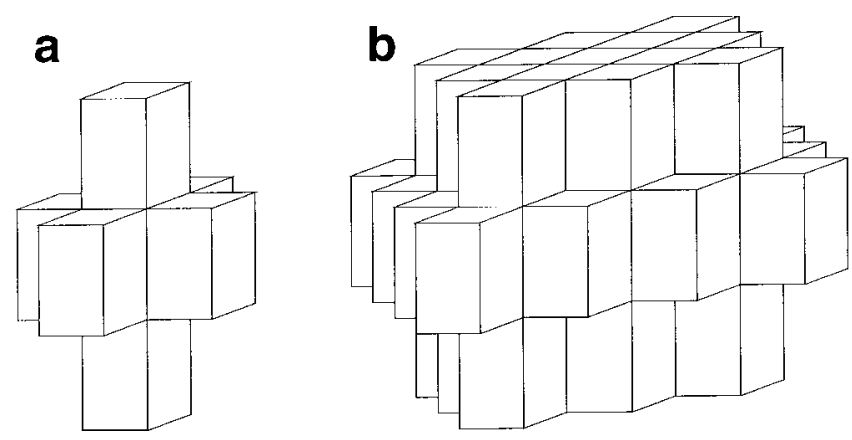

Figure 2.

a: Structuring element obtained by thresholding an isotropic distance map would have the same radius, measured in voxels, in all directions (here 3 voxels). Using this element does not yield isotropic erosion. b: In contrast, thresholding our anisotropic distance map yields a much more spherical structuring element than in (a) for the same 3-voxel height. Morphological erosion with the element (b) hence does not favor any particular direction, and makes the erosion more insensitive to voxel geometry.

may not look spherical if the distance used is not isotropic. This is the case when the distance is expressed in units of voxels and the voxels are not cubic. We consequently devised a new anisotropic chamfer distance map (ACDM; detailed in Surface Matching, below) to obtain truly isotropic erosion. This improvement yields more reliable erosion for data sets with large slice thickness because it erodes approximately the same actual volume in all directions, rather than the same number of pixels (Fig. 2), making the method insensitive to voxel geometries. In our implementation, the erosion size defaulted to the longest diagonal voxel size in the data set processed; this proved sufficient in most cases to fully disconnect the brain from surrounding tissues. Isolation of the eroded binary brain from the remaining parasitic masses (eroded tongue, etc.) was achieved by an 8-connected 3D flooding of the brain, starting from the centroid of the eroded volume. Because this centroid might fall into a cavity in the eroded volume, such as the ventricles, a 3D cross was automatically drawn to connect it to the binary brain.

After erosion and extraction of the binary brain, a 3D binary morphological dilation [Schmitt and Mattioli, 1994] of the extracted brain was applied to recover its original size. Morphological dilation, which is the dual operation to erosion, can be intuitively interpreted as an inflation of the objects in an image by a fixed size. Fast 3D dilation by a solid sphere with slightly larger radius than the one used for erosion was obtained by thresholding an ACDM. Since peripheral objects have been eliminated in the previous step, only the brain is dilated. The resulting brain shape is smooth, which is characteristic of morphological dilation, and larger than the actual brain by 1-2 $\mathrm{mm}$. Brain shape refinement was hence applied by masking the dilated volume with the first thresholded binary volume, in which most brain contours already existed among many other contours. The effect of this masking is two-fold: in regions where brain contours are present in the first thresholded volume, they are exactly recovered and the dilated brain size is sufficiently small not to include unwanted meninges and muscle; in regions where no contours are present in the first thresholded volume (typically reigons where even human observers have difficulty defining the brain surface), the actual brain shape is approximated by the dilated shape. Cavities in the brain were then flooded, and the final detection of the marginal contours of the refined brain was performed by a simple binary $2 \mathrm{D}$ contour extraciton algorithm in every slice. This method is preferable to $3 \mathrm{D}$ contour detection when using thick slices (5-20 mm), because it always yields accurate 1-pixel-wide contours even when the brain shape changes rapidly between adjacent slices. The contours were finally converted to a set of $3 \mathrm{D}$ vertices (3 coordinates per point) for interactive real-time 3D display. A visual summary of this method is presented in Figure 1 for a patient with a gross structual abnormality. Our brain segmentation method can be used for axial, sagittal, and coronal orientations.

The erosion and dilation steps are not necessary for HMPAO SPECT images, which show no significant peripheral uptake close to the brain. No SPECT histogram interpretation was necessary, since full SPECT brain surface extraction is achieved in only a few seconds, making manual threshold adjustments fast and easy. The lower and higher thresholds simply default to $45 \%$ and $100 \%$ of the maximum voxel count for xenon-calibrated HMPAO SPECT; the method is fully automatic in most cases. SPECT slices may be discarded if they are blurred or outside the brain.

\section{Surface matching}

For the registration of two different data sets, the data set with higher spatial resolution is usually chosen as a fixed reference for the other (mobile) data set. The previously extracted volume of brain contours is used for the reference brain surface, while the previously extracted 3D vertices are used for the mobile surface. We present here the general surfacematching algorithm; specific applications are discussed in the next section.

Reference brain contours first undergo a contours interpolation to create a three-dimensionally coherent 
set of contours with high accuracy: a new slice is created between two adjacent original slices, containing the interpolated transition between the contours in the slices immediately above and below. The resulting brain shape is hence fully 3D coherent even when the brain sections vary largely between adjacent slices. It is also more accurate than the result of a $3 \mathrm{D}$ edge detection because the slice thickness in the enhanced volume is half the original one. The contour interpolation is achieved by flooding and logical operations.

Interpolated reference brain contours are then transformed into an ACDM. Such a map approximates the Euclidean distance, but is computed considerably more quickly (linear complexity). We extended the $3 / 4 / 5$ chamfer distance transform as presented by Borgefors [1986] by using an anisotropic $3 \times 3 \times 3$ elementary distance pattern. With this approach, scaled approximations of the real Euclidean distances between one voxel and its 26 adjacent 3D neighbors are used instead of the values 3 (for straight neighbors), 4 (for diagonal neighbors in the same plane), and 5 (for diagonal neighbors in adjacent planes). This enhancement was devised to account for elongated parallelepipedic voxels. Distances are stored in the ACDM as 8-bit values, and loss of precision is avoided by the following scaling: the elementary distance between diagonal voxels in two adjacent slices is scaled to the value 50; the same scale factor is applied to the other elementary distances in the $3 \times 3 \times 3$ pattern, and the results are rounded to the closest integer value (Fig. 3).

Mobile surface data points, which are stored as 3D vertices, are modified according to a global rigid transformation with up to 9 parameters: 3 rotations, 3 translations, and 3 scale factors. Scaling should not be used if the voxel dimensions are known precisely from the imaging devices. Scaling parameters can easily be disabled in our method. In a first step, the first-order moments, i.e., centers of mass, of the reference and mobile surfaces are matched. This is used as a fast automatic method for computing an appropriate starting point for subsequent surface matching. The matching error between transformed mobile vertices and reference contours is then evalated as a function (generalized distance) of the values read in the distance map at the locations of the transformed vertices. This general method is known as a chamfer-matching technique [Barrow et al., 1977; Mangin et al., 1994; van Herk and Kooy, 1994].

In order to allow the robust registration of partial brain volumes, we propose a new definition for the generalized distance D. Given a reference volume of brain contours bounded in the $\mathrm{x}, \mathrm{y}$, and $\mathrm{z}$ directions by

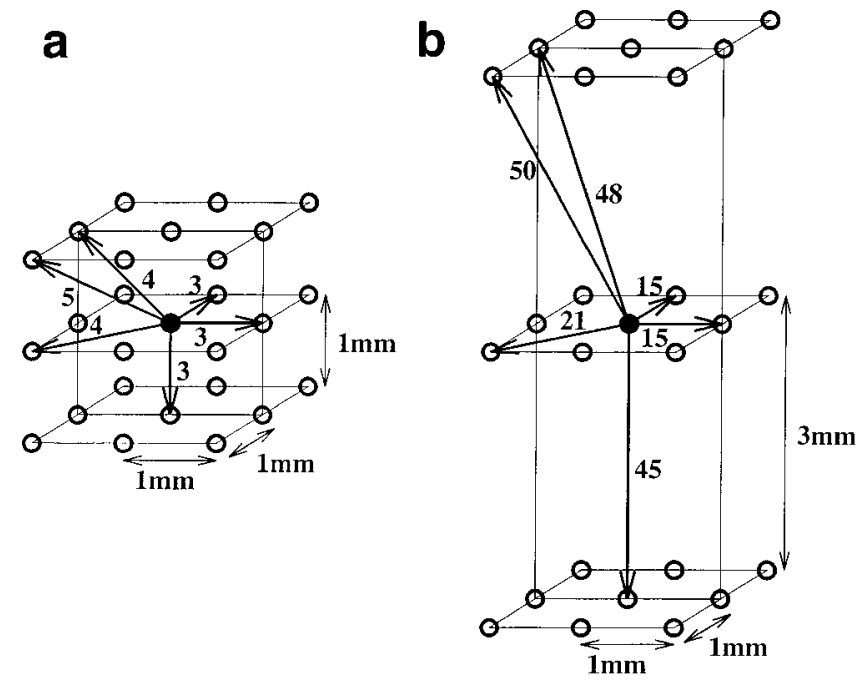

Figure 3.

The 3/4/5 chamfer distance transform (a) was originally designed for isotropic lattices, but is inadequate for data sets in which the slice thickness is larger than the in-slice resolution (b). Better approximations of the real Euclidean distance are obtained in (b) with our anisotropic chamfer distance transform. The largest Euclidean distance between the center location and one of its neighbors is scaled to 50; the same scale factor is then applied in the elementary $3 \times 3 \times 3$ lattice to all the other Euclidean distances to the center.

$\left(x_{\min }, x_{\max }\right),\left(y_{\min }, y_{\max }\right)$, and $\left(z_{\min }, z_{\max }\right)$, and a mobile surface (a set of vertices) $S_{\mathrm{Mob}}$, the generalized distance is computed as the root mean square of individual point-to-contour distances, evaluated differently for two subsets of $S_{\text {Mob }}\left(S_{1}\right.$ and $S_{2}$ defined below):

$$
\mathrm{D}=\frac{1}{\operatorname{card}\left(\mathrm{S}_{1}\right)+\operatorname{card}\left(\mathrm{S}_{2}\right)} \sqrt{\left(\sum_{\mathrm{p} \in \mathrm{S}_{1}} \mathrm{~d}_{\mathrm{ref}}^{2}(\mathrm{p}) \mid+\operatorname{card}\left(\mathrm{S}_{2}\right) \mathrm{d}_{\max }^{2}\right.}
$$

with card(S) being the number of elements in a set $S$ and:

$\left\{\begin{array}{l}\mathrm{S}_{1}=\left\{\mathrm{p} \in \mathrm{S}_{\mathrm{Mob}} ; \mathrm{z}_{\min } \leq \mathrm{z}_{\mathrm{p}} \leq \mathrm{z}_{\max } ; \mathrm{y}_{\min } \leq \mathrm{y}_{\mathrm{p}} \leq \mathrm{y}_{\max } ; \mathrm{x}_{\min } \leq \mathrm{x}_{\mathrm{p}} \leq \mathrm{x}_{\max }\right\} \\ \mathrm{S}_{2}=\left\{\mathrm{p} \in \mathrm{S}_{\mathrm{Mob}} ; \mathrm{z}_{\min } \leq \mathrm{z}_{\mathrm{p}} \leq \mathrm{z}_{\max } ; \mathrm{y}_{\mathrm{p}}<\mathrm{y}_{\min }\left|\mathrm{y}_{\mathrm{p}}>\mathrm{y}_{\max } ; \mathrm{x}_{\mathrm{p}}<\mathrm{x}_{\min }\right| \mathrm{x}_{\mathrm{p}}>\mathrm{x}_{\text {max }}\right\}\end{array}\right.$

where the $(x, y)$ plane is the reference slice plane and $\mathrm{d}_{\mathrm{ref}}(\mathrm{p})$ is the distance (read in the distance map with trilinear interpolation) between a point $p$ in the transformed mobile surface $S_{\mathrm{Mob}}$ and the reference brain contours. Points in $S_{1}$ are within the reference volume boundaries $\left(\mathrm{x}_{\min }, \mathrm{x}_{\max } ; \mathrm{y}_{\min }, \mathrm{y}_{\max } ; \mathrm{z}_{\min }, \mathrm{z}_{\max }\right)$ and yield conventional point-to-contours distance measurements. Points in $S_{2}$ (outside the reference bounds in $x$ or y but within bounds in $\mathrm{z}$ ) yield the maximal possible distance $d_{\max }(255$ in our implementation) because the head of the subject should remain within the $x$ and $y$ 
reference slice boundaries. On the contrary, points in $S_{\mathrm{Mob}}$ but neither in $S_{1}$ nor in $S_{2}$ (outside the reference bounds in $\mathrm{z}$, but not in $\mathrm{x}$ nor $\mathrm{y}$ ) are simply undetermined since there are no reference slices at those particular locations. Hence, these points do not contribute to the generalized distance, allowing for registration of partial brain volumes. Because the number of points participating in the calculation of $\mathrm{D}$ varies with the position and orientation of the mobile surface, $\mathrm{D}$ is not continuous. This prevents the use of conventional gradient-based minimization techniques.

Powell's minimization algorithm for nonregular functions [Powell, 1964; Pelizzari et al., 1989; Press et al., 1992; van Herk and Kooy, 1994] is consequently used as a local automatic matching method to minimize the distance between both surfaces. This algorithm consists of iterative uses of a minimization algorithm for one-dimensional functions along cleverly chosen vectors (directions) in the multidimensional parameter space. Its power resides in the use of linear combinations of the provided parameters as search directions. This allows the algorithm to find minima in regions of the 9D search space where searches along orthogonal directions would not lead.

Like all local algorithms, Powell's algorithm might converge to a local minimum of the arbitrarily shaped distance function, instead of finding the global minimum. Therefore, we devised specific semiautomatic supervision tools to correct for possible false convergences. Manually chosen 2D sections of the 9D distance hypersurface show the evolution of the generalized distance with the selected parameter $( \pm 20 \mathrm{~mm}$ for translation parameters, $\pm 20^{\circ}$ for rotation parameters, or \pm 0.1 for scaling factors). If necessary, the parameter value yielding the minimum distance in the $2 \mathrm{D}$ section may be transferred automatically to the corresponding starting parameter value for Powell's algorithm to find the next closest local minimum. This simple supervision scheme also solves, with minimal user interaction, the problem of the initial relative positioning of partial brain scans, for which first-order moment matching might not yield a satisfactory starting point. The success of the surface matching can also be checked visually in fast low-resolution $2 \mathrm{D}$ reslicing. Isocontours extracted from the reference data set are displayed on the corresponding mobile image to check for internal structures as well as brain contours. Real-time 3D display of both brain surfaces with mouse-driven translation, rotation, and zooming also allows inspection of the registration accuracy and immediate detection of false convergences.

Finally, our method includes the possibility of eliminating all the points in the mobile surface that are farther than a specified distance from the reference surface (outliers) [Mangin et al., 1994]. This step was usually applied after an initial coarse surface matching. It was included to account for differences in brain shapes from functional and structural imaging modalities (e.g., focal cortical hypoperfusion), and can be introduced during matching to obtain a more precise final convergence. Its effects can be observed in the 2D distance sections: small local minima are eliminated and the slope of the distance function around the global maximum is increased.

After convergence to the surface-matching process, the mobile data set was resliced to the orientation and spatial resolution of the reference data set using trilinear interpolation. The original 16-bit image intensities were used for reslicing, in order to avoid the potential loss of quantitative accuracy resulting from the conversion to 8-bit performed prior to image segmentation.

\section{Applications}

Our method has been successfully applied to the registration of MRI, fMRI, pMRI, MRS, and SPECT scans (Figs. 4,5). The registration of pMRI or fMRI with other functional modalities such as SPECT is performed through two successive registrations. First, the SPECT volume is registered with a structural MR localizer volume. Second, this structural MRI is registered with the fMRI or pMRI, using the relative positioning known from the scanner as a starting point. This second registration corrects for possible small head motion between the structural and functional MR scans. The first structural view from the functional time sequences (e.g., one of the 40 sequentially scanned MR images forming a single-slice pMRI map) is used for registration, rather than the postprocessed z-score or perfusion maps.

All six rotation and translation parameters are used for registration of multislice fMRI and MRI, since the starting point provided by the MR scanner is usually quite accurate, making the registration process unlikely to diverge. Contrarily, only the two translations within the pMRI slice plane and the rotation about the axis orthogonal to this plane are used for registration of pMRI and MRI, because coregistration of a plane and a volume is particularly ill-posed. We hence neglect head shifts along the axial direction between the MRI and subsequent pMRI acquisitions, while we account for left- or right-sided head tilts. The possibility of head motion during the functional time sequence acquisition, currently being studied in our laboratory, is not addressed here. 


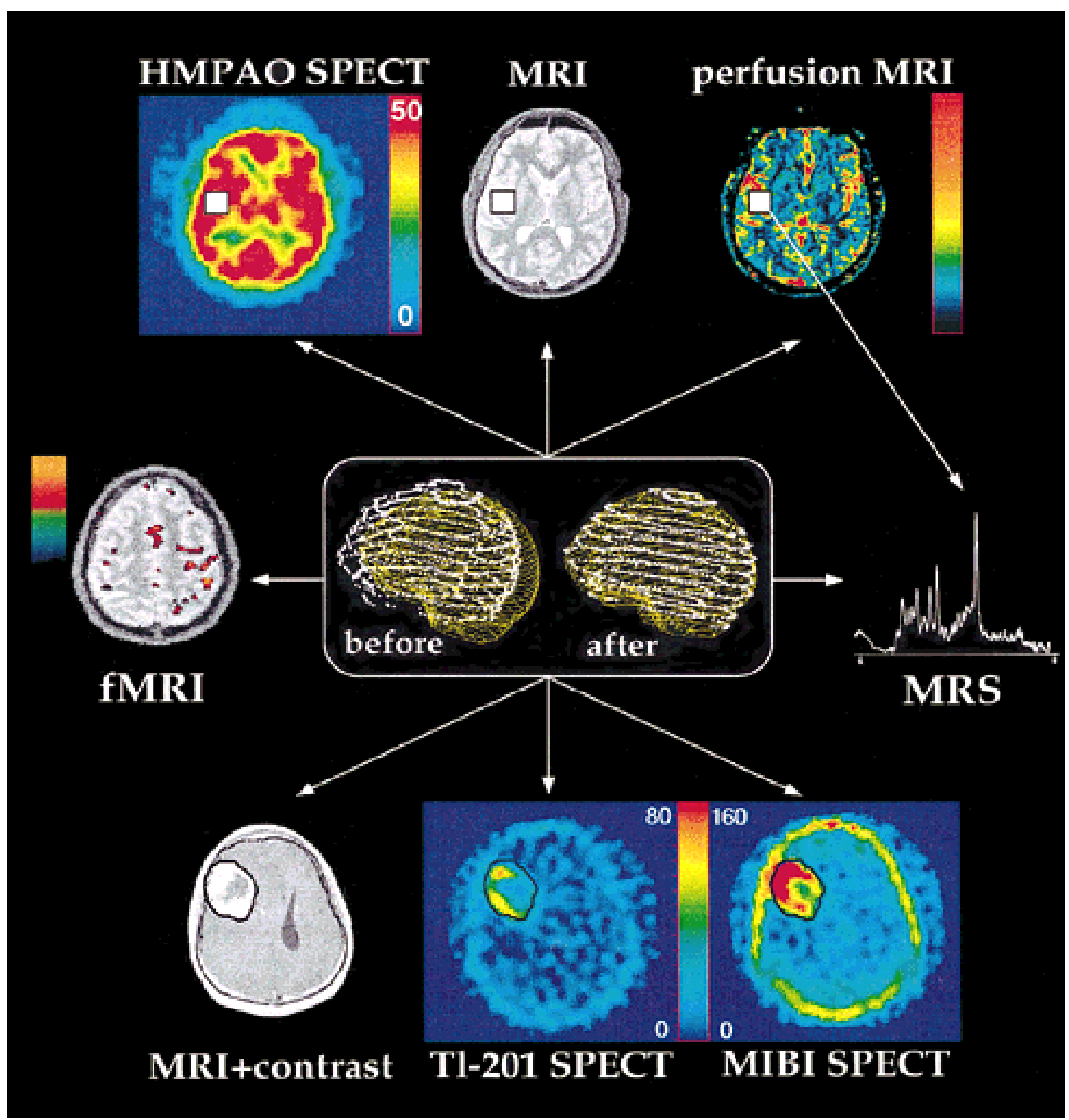

Figure4.

Applications of our registration method to a normal volunteer (top ence MRI, using 3D rotations and translations; MR spectroscopy and middle) and to a tumor patient (bottom). Top: An MRI localizer (middle) was used as fixed reference for registration of xenon-calibrated HMPAO SPECT, using 3D rotations and translations (left), and perfusion MRI, using the two translations and one rotation in the pMRI plane (right). Middle: Functional MRI (left; right hand finger-tapping task) was registered to the same refer-

(right), naturally aligned with the reference MRI, could be correlated to pMRI and SPECT. Bottom: MRI, 201-TI SPECT, and MIBI SPECT could be registered without manual adjustments during surface matching, despite anatomical and technical abnormalities ( 3 blurred slices in each SPECT (not shown), and slight ghost artifacts in MRI). 


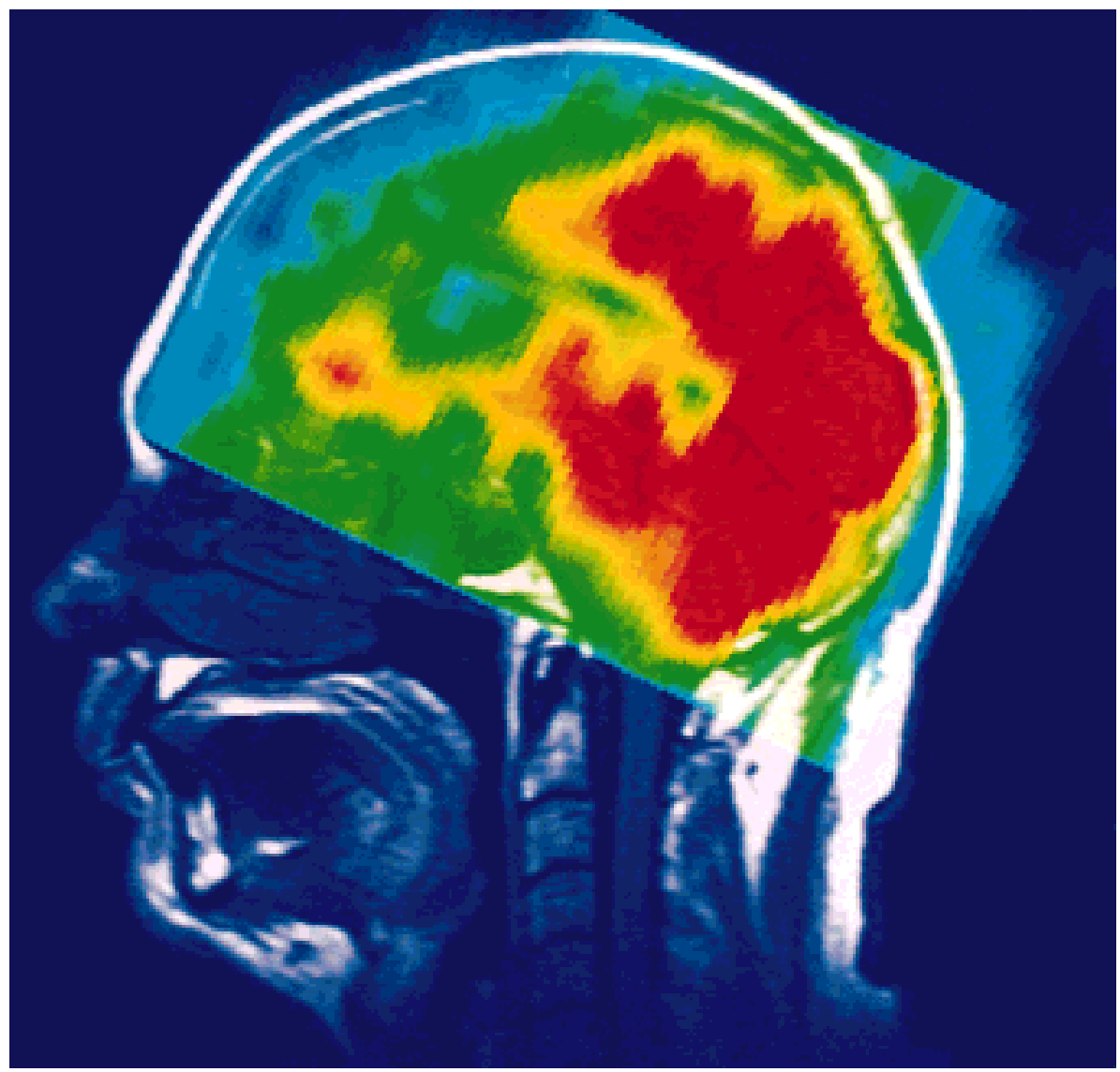

Figure 5

Superimposed visualization of registered $\mathrm{T}_{1}$-weighted $\mathrm{MRI}$ ( $T E=8 \mathrm{msec}$, TR $=500 \mathrm{msec}$,

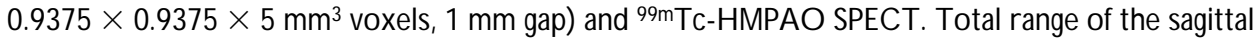
MRI is $6.6 \mathrm{~cm}$ (11 slices), and the axial SPECT shows severe frontal hypoperfusion. No manual adjustment was performed.

For registration of MRS voxels with functional volumes, such as SPECT, registration is first performed between SPECT and the 3D MRI localizer for the spectroscopy voxels. The coordinates of the MRS voxels, known from the MR scanner in the referential of the MRI localizer, are then directly known in the referential of the reconstructed SPECT. Using the MRS voxel size information provided by the MR scanner, a $3 \mathrm{D}$ region of interest is created for accurate quantitative measures from SPECT and MRI within the MRS voxel.

\section{Validation}

The validity of our process was assessed with simulations, and the combined accuracy of registration and drawing of regions of interest was assessed with images from two clinical populations. Validation was performed with MRI and SPECT registration, since it is our most widely used application, and the most difficult because of the low resolution and contrast of SPECT.

The first part of our validation addresses the accuracy and robustness of the proposed procedure using MRI and simulated SPECT images. In the following, a "transformation" means three rotations of $\pm 5- \pm 15^{\circ}$ and three translations of $\pm 5- \pm 15 \mathrm{~mm}$, performed by one of the authors (L.C.) and recovered blindly by another (L.I.) Eleven different transformations were used. In order to allow accurate SPECT simulations through $3 \mathrm{D}$ convolutions, an axial $\mathrm{T}_{1}$-weighted contiguous MRI covering the whole head and neck was acquired (TE $=8 \mathrm{msec}$, $\mathrm{TR}=500 \mathrm{msec}$, $0.9375 \times 0.9375 \times 5 \mathrm{~mm}^{3}$ voxels, 51 slices). The accu- 
racy of the surface-matching procedure and ACDM was evaluated by automatically extracting the brain surface of this volume, truncated to our usual scanning size ( 26 slices, from the bottom of the cerebellium to 1 $\mathrm{cm}$ from the top of the skull), and by matching this surface to a transformed version of itself. Additional errors due to reslicing the MRI surface extraction were then investigated by reslicing the truncated MRI to $0.9375 \times 0.9375 \times 5 \mathrm{~mm}^{3}$ voxels according to a given transformation, automatically extracting its surface (no manual adjustment), and matching it to the original truncated MRI. Additional errors due to low SPECT resolution were then evaluated by removing the fat from the full original volume by simple tresholding, reslicing it according to a given transformation, convolving it by a separable 3D Gaussian of $12.4 \times 12.4 \times 20 \mathrm{~mm}^{3}$ FWHM, subsampling it to $3.3 \times 3.3 \times 20 \mathrm{~mm}^{3}$ voxels, automatically extracting its surface using our SPECT segmentation method, and matching it to the original truncated MRI. Since we are only interested in extracting the SPECT brain surface, it was not necessary to remap the MRI gray/white matter intensities to typical SPECT counts; however, eliminating the fat from the $\mathrm{T}_{1}$-weighted MRI prior to convolution was necessary to avoid spurious SPECT active spots in the fatty regions of the head. Additional errors due to noise in SPECT acquisitions were evaluated by adding strong Poisson noise independently to each voxel of the previous simulated SPECT (variance equal to intensity of a given voxel) and repeating the previous steps. Finaly, additional errors due to anatomical-functional shape differences were evaluated by truncating the right frontal lobe of the noisy simulated SPECT by an ellipsoid with diameters of $10 \times 20 \times 40$ $\mathrm{mm}$ and repeating the previous steps.

In order to evaluate the combined accuracy of the registration method, imaging modalities, and manual drawing of regions of interest with clinical data sets, MIBI SPECT vs. MRI + gadolinium registration was performed in 6 brain tumor patients. MIBI images are particularly difficult to register because of their low contrast and because there is almost no uptake in normal brain tissue [Chiu et al., 1990]. Images were registered using automatic brain surface extraction for MRI (some manual threshold adjustments were required for SPECT) and the automatic surface-matching method. The eyeballs were visible in MIBI SPECT as hypoactive spots, and the choroid plexus of the lateral ventricles showed high MIBI uptake. These three structures were outlined independently in both SPECT and MRI, and distances between the centroids of these landmarks were measured.
HMPAO SPECT and MRI from 10 subjects (5 normals and 5 patients with advanced frontotemporal dementia) were registered using the same analysis procedure as for the MIBI study. The five pathological studies were chosen to evaluate the robustness of the registration method for subjects with very large regions of hypoperfusion in SPECT. Most of the extent of their frontal lobes showed blood flow below $40 \%$ of the blood flow measured in the primary visual cortices. Because the shapes of internal brain structures seen in SPECT do not correlate accurately with their anatomical counterparts (see the shapes of the basal ganglia and of the thalami in the top row of Fig. 4), we used three other objective anatomical landmarks visible in both MRI and SPECT: the centers of the eyeballs and the most medial point of the posterior section of the superior sagittal sinus (SSS). Although it lies on the brain's surface, the SSS was used because of the lack of any other objective MRI and SPECT landmark in the orbito-meatal plane. Triangles connecting these three points were drawn independently on MRI and SPECT, on the highest and lowest slices in which they were visible. Comparisons of the triangles from SPECT and MRI allowed measurements of real 3D dismatch. The distance between the centroids of the triangles and the maximal distance between corresponding vertices from both triangles were computed as a measure of accuracy. This validation was designed to check all the registration parameters.

\section{RESULTS}

The multimodality registration method presented has been used with a variety of SPECT agents, and MRI sequences and orientations, and has never failed after more than 350 registrations performed. Table I shows a representative sample of the global processing time for MRI and SPECT registration with normal subjects and advanced brain degeneration patients. These times were measured externally and, for a multitasking networked system, are about twice the actual processing times used by our algorithms. The total registration time in this sample varied from 6-11 min, depending on the size of the data sets and the specific problems associated with each imaging study. Registration of the other images presented above (pMRI, fMRI) was generally easier due to the higher spatial resolution of these data sets, and usually did not require user interaction.

Results of the simulations are presented in Table II. Matching a surface to a transformed version of itself yielded accuracies of the order of the convergence criterion of Powell's algorithm (1.0E-7 in our implemen- 
TABLE I. MRI/HMPAO SPECT registration times determined for a representative sample of normal subjects, and for patients with Alzheimer's disease and fronto-temporal dementia*

\begin{tabular}{|c|c|c|c|c|c|c|c|c|c|c|c|c|c|}
\hline \multirow[b]{3}{*}{ Patient } & \multicolumn{2}{|c|}{ MRI exam } & \multirow{2}{*}{\multicolumn{2}{|c|}{ Convert to AVS }} & \multirow{2}{*}{\multicolumn{4}{|c|}{ Extract surfaces }} & \multirow{2}{*}{\multicolumn{2}{|c|}{ Match }} & \multirow{3}{*}{$\begin{array}{c}\text { Reslice, } \\
2 \text { ways } \\
\text { (min:sec) }\end{array}$} & \multirow{3}{*}{$\begin{array}{c}\text { Residual } \\
\text { distance } \\
(\mathrm{mm})\end{array}$} & \multirow{3}{*}{$\begin{array}{c}\text { Total } \\
\text { time } \\
\text { (min:sec) }\end{array}$} \\
\hline & \multirow{2}{*}{$\begin{array}{l}\text { Thick- } \\
\text { ness } \\
(\mathrm{mm})\end{array}$} & \multirow[b]{2}{*}{$\begin{array}{l}\text { Slices } \\
\text { (no.) }\end{array}$} & & & & & & & & & & & \\
\hline & & & $\begin{array}{c}\text { MRI } \\
\text { (min:sec) }\end{array}$ & $\begin{array}{c}\text { SPECT } \\
\text { (min:sec) }\end{array}$ & $\begin{array}{c}\text { MRI } \\
\text { (min:sec) }\end{array}$ & $\begin{array}{c}\text { IC } \\
\text { (no.) }\end{array}$ & $\begin{array}{c}\text { ACDT } \\
\text { (min:sec) }\end{array}$ & $\begin{array}{c}\text { SPECT } \\
\text { (min:sec) }\end{array}$ & (min:sec) & $\begin{array}{l}\mathrm{IC}^{\mathrm{a}} \\
\text { (no.) }\end{array}$ & & & \\
\hline \multicolumn{14}{|c|}{ Axial proton density } \\
\hline Normal 1 & 5.0 & 26 & $0: 45$ & $0: 25$ & $1: 16$ & 0 & $0: 56$ & $0: 45$ & $2: 24$ & 0 & $1: 16$ & 1.66 & 08:47 \\
\hline Normal 2 & 5.0 & 29 & $0: 50$ & $0: 25$ & $1: 30$ & 0 & 1:01 & $0: 39$ & $2: 16$ & 0 & $1: 21$ & 1.76 & 09:02 \\
\hline Normal 3 & 5.0 & 26 & $0: 45$ & $0: 25$ & $2: 34$ & 1 & $0: 58$ & $0: 47$ & $1: 45$ & 0 & $1: 13$ & 1.50 & $09: 27$ \\
\hline $\mathrm{AD} 1$ & 7.5 & 18 & $0: 40$ & $0: 25$ & $0: 58$ & 0 & $0: 38$ & $0: 43$ & $2: 37$ & 0 & $0: 52$ & 1.64 & $07: 53$ \\
\hline AD 2 & 5.0 & 25 & $0: 45$ & $0: 25$ & $4: 13$ & 3 & $0: 55$ & $0: 31$ & $1: 36$ & 0 & $1: 10$ & 1.87 & $10: 35$ \\
\hline $\mathrm{AD} 3$ & 5.0 & 26 & $0: 45$ & $0: 25$ & $1: 26$ & 0 & $0: 56$ & $0: 51$ & $1: 32$ & 0 & $1: 14$ & 1.69 & 08:09 \\
\hline $\mathrm{AD} 4$ & 5.0 & 29 & $0: 50$ & $0: 25$ & $1: 30$ & 0 & $0: 59$ & $0: 34$ & $2: 05$ & 0 & $1: 22$ & 1.73 & $08: 45$ \\
\hline AD 5 & 5.0 & 28 & $0: 50$ & $0: 25$ & $1: 29$ & 0 & $0: 58$ & $1: 12$ & $3: 38$ & 3 & $1: 20$ & 1.86 & $10: 52$ \\
\hline FTD 1 & 7.5 & 17 & $1: 45$ & $0: 25$ & $0: 53$ & 0 & $0: 36$ & $0: 37$ & $1: 44$ & 0 & $0: 49$ & 1.94 & $07: 49$ \\
\hline FTD 2 & 5.0 & 26 & $0: 45$ & $0: 25$ & $1: 19$ & 0 & $0: 57$ & $0: 48$ & $2: 56$ & 1 & $1: 19$ & 2.05 & 09:29 \\
\hline \multicolumn{14}{|c|}{ Sagittal T1-weighted } \\
\hline AD 6 & 6.0 & 11 & $0: 35$ & $0: 25$ & $1: 10$ & 1 & $0: 23$ & $0: 31$ & $1: 55$ & 0 & $0: 36$ & 1.98 & $06: 35$ \\
\hline AD 7 & 6.0 & 11 & $0: 35$ & $0: 25$ & 1:01 & 1 & $0: 22$ & $0: 51$ & $1: 45$ & 0 & $0: 38$ & 1.87 & $06: 37$ \\
\hline AD 8 & 6.0 & 11 & $0: 35$ & $0: 25$ & $0: 38$ & 0 & $0: 22$ & $0: 34$ & $1: 50$ & 0 & $0: 35$ & 1.65 & $05: 59$ \\
\hline AD 9 & 6.0 & 11 & $0: 35$ & $0: 25$ & 2:00 & 2 & $0: 23$ & $1: 12$ & $2: 29$ & 1 & $0: 38$ & 1.84 & $08: 42$ \\
\hline FTD 3 & 5.0 & 17 & $0: 40$ & $0: 25$ & $1: 31$ & 1 & $0: 35$ & $0: 41$ & $1: 34$ & 0 & $0: 45$ & 1.83 & $07: 11$ \\
\hline \multicolumn{3}{|c|}{ AVS program load time } & 0:05 & 0:05 & $0: 15$ & & & $0: 10$ & $0: 25$ & & $0: 10$ & & 01:10 \\
\hline Minimum & & 11 & $0: 35$ & $0: 25$ & $0: 38$ & 0 & $0: 22$ & $0: 31$ & $1: 32$ & 0 & $0: 35$ & 1.50 & $05: 59$ \\
\hline Maximum & & 29 & $1: 45$ & $0: 25$ & $4: 13$ & 3 & $1: 01$ & $1: 12$ & $3: 38$ & 3 & $1: 22$ & 2.05 & $10: 52$ \\
\hline Average & & 21 & $0: 47$ & $0: 25$ & $1: 34$ & 0.6 & $0: 44$ & $0: 45$ & 2:08 & 0.3 & 1:01 & 1.79 & $08: 23$ \\
\hline Standard & eviation & 7 & $0: 16$ & 0:00 & $0: 50$ & 0.9 & $0: 15$ & $0: 12$ & $0: 34$ & 0.8 & $0: 18$ & 0.14 & $01: 22$ \\
\hline
\end{tabular}

*AD, Alzheimer's disease; FTD, fronto-temporal dementia.

${ }^{a} \mathrm{IC}=$ number of interactive corrections needed (each consists of one intuitive mouse-driven modification of one parameter).

b Total time includes an extra 1:00 for patient and menu selection by unexperienced users.

tation; see Press at al. [1992] and demonstrated the accuracy of our ACDM-based surface-matching procedure. Errors are larger for Z-translations because of the larger voxel size in this direction $(5 \mathrm{~mm}$ slice thickness). After reslicing to MRI resolution, automatic surface extraction, and matching, the errors obtained were approximately 10 times smaller than imaging resolution (to compare rotation and translation errors, we note that a rotation of $1^{\circ}$ would yield a translation of $1.75 \mathrm{~mm}$ at $10 \mathrm{~cm}$ from the center). After blurring of the transformed MRI volumes, the errors remained approximately 10 times smaller than SPECT resolution, with the exception of Z-translations, probably because only 11 SPECT slices did not provide sufficient information in this direction. Addition of noise to the simulated SPECTs did not uniformly degrade the accuracy of the registration, which suggests that our simple method for extracting SPECT surfaces is adequate. The introduction of a large ellipsoidal defect finally added errors far below SPECT resolution, and the parameters recovered with and without the defect were identical with submillimetric accuracy. This sug- gests that our elimination of outliers was efficient in matching surfaces in the presence of anatomicalfunctional shape differences.

Results of the MIBI study (Table III) show that the maximum distance between the centroids of the triangles drawn was in the order of the SPECT resolution (12.4 mm FWHM). Several slices were blurred in studies 2,4 , and 6 by patient motion. In addition, the MRI of patient 4 (presented in the bottom row of Fig. 4) was slightly blurred. Tumors extending through the cortex yielded dissimilar brain shapes in SPECT and MRI (patients 2, 4, and 6; see Fig. 4 for patient 4) because of different uptake mechanisms for MIBI [Chiu et al., 1990] and gadolinium [Hasso, 1993]. Despite these difficulties, the average combined accuracy of the registration procedure and the drawing of small regions on SPECT and MRI was approximately $5 \mathrm{~mm}$.

The HMPAO study (Table IV) indicates that the maximal error in the localization of a precise structure in SPECT using registered MRI was on the order of the SPECT resolution. The mean combined registration 
TABLE II. Registration accuracy assessed using MRI $\left(0.937 \times 0.9375 \times 5 \mathrm{~mm}^{3}\right.$ voxels; 51 slices used for simulation of SPECT through convolution, 26 slices used for surface matching) and simulated SPECT (12.4 mm in-plane FW HM, 20 mm slice thickness; 11 nonuniformly spaced slices with $3.3 \times 3.3 \times 20 \mathrm{~mm}^{3}$ voxels)*

\begin{tabular}{|c|c|c|c|c|c|c|c|}
\hline & & $\begin{array}{c}\text { Rotation-X } \\
\text { (degrees) }\end{array}$ & $\begin{array}{c}\text { Rotation-Y } \\
\text { (degrees) }\end{array}$ & $\begin{array}{c}\text { Rotation-Z } \\
\text { (degrees) }\end{array}$ & $\begin{array}{c}\text { Transl-X } \\
(\mathrm{mm})\end{array}$ & $\begin{array}{l}\text { Transl-Y } \\
(\mathrm{mm})\end{array}$ & $\begin{array}{c}\text { Transl-Z } \\
\text { (mm) }\end{array}$ \\
\hline \multirow[t]{2}{*}{ 1) MRI surface vs. itself displaced } & Average & $1.38 \mathrm{E}-06$ & $6.86 \mathrm{E}-07$ & 7.87E-07 & $6.50 \mathrm{E}-07$ & $3.71 \mathrm{E}-07$ & $1.23 \mathrm{E}-06$ \\
\hline & Maximum & $1.65 \mathrm{E}-06$ & $2.55 \mathrm{E}-06$ & $1.40 \mathrm{E}-06$ & 2.23E-06 & $1.23 \mathrm{E}-06$ & 2.10E-06 \\
\hline \multirow[t]{2}{*}{ 2) MRI vs. displaced MRI } & Average & 0.07 & 0.10 & 0.04 & 0.02 & 0.06 & 0.49 \\
\hline & Maximum & 0.17 & 0.25 & 0.10 & 0.04 & 0.30 & 0.60 \\
\hline \multirow[t]{2}{*}{ 3) MRI vs. blurred displaced MRI } & Average & 1.14 & 0.50 & 0.91 & 0.50 & 1.12 & 3.79 \\
\hline & Maximum & 2.39 & 1.26 & 1.66 & 2.14 & 2.75 & 5.84 \\
\hline \multirow[t]{2}{*}{ 4) MRI vs. blurred, noisy, displaced MRI } & Average & 1.13 & 1.58 & 1.29 & 0.67 & 1.03 & 3.15 \\
\hline & Maximum & 2.47 & 2.67 & 2.87 & 2.38 & 2.67 & 5.56 \\
\hline \multirow{2}{*}{$\begin{array}{l}\text { 5) MRI vs. blurred, noisy, truncated, } \\
\text { displaced MRI }\end{array}$} & Average & 1.45 & 1.93 & 1.64 & 0.64 & 1.16 & 3.22 \\
\hline & Maximum & 3.91 & 3.81 & 3.44 & 2.57 & 3.04 & 5.66 \\
\hline \multirow{2}{*}{$\begin{array}{l}\text { 6) Difference between parameters in } \\
\text { 4) and 5) }\end{array}$} & Average & 0.50 & 0.57 & 0.57 & 0.31 & 0.21 & 0.17 \\
\hline & Maximum & 1.44 & 2.46 & 1.39 & 0.83 & 0.47 & 0.84 \\
\hline
\end{tabular}

* Anatomical-functional differences have been introduced (strong uncorrelated noise with variance equal to the mean of each SPECT voxel, truncation of one frontal lobe by an ellipsoid with $10 \times 20 \times \times 40 \mathrm{~mm}$ diameter) in order to assess the accuracy of our surface extraction and registration methods with difficult data sets.

and drawing error was $<4 \mathrm{~mm}$, despite the extremely low uptake in the frontal lobes of patients 8,9 , and 10 .

In all difficult cases studied, the interactive correction methods proved useful to quickly overcome atypical difficulties. For example, no more than $10-20$ sec of interaction were needed during supervision of the surface-matching algorithm, although we observed initial angles as large as $35^{\circ}$ and translations as large as $40 \mathrm{~mm}$.

\section{DISCUSSION}

Our registration method has been optimized to increase the efficiency and robustness of surfacematching methods, particularly with difficult data sets. All registration steps are automatic, but quick and easy manual adjustments are available for difficult studies, making the method more robust with atypical cases.

Automatic surface extraction is up to 20 times faster than manual drawing (1 min, $40 \mathrm{sec}$ for 30 slices, vs. 1 min at best per slice in manual methods). Automatic MRI brain surface extraction was achieved for all the studies regardless of orientation, technical quality, and anatomical abnormalities. Manual drawing or editing of brain contours was never needed, and no tool has been implemented for this function. The fully 3D morphological approach allows surface extraction from a single set of images, whereas more classical clustering methods usually requie multiecho datasets [Ardekani et al, 1994]. Our automatic histogram interpretation eliminated in most cases the need for interactive threshold adjustments reported previously [Mangin et al., 1994; Alpert et al., 1996]. Because different MR sequences yield highly variable histogram shapes, the automatic interpretation may fail with unusual MR sequences. However, it rarely fails with the protondensity MR sequence, for which it was originally designed. The flat histograms of some images would also cause failure in modal histogram analysis methods [DeCarli et al., 1992]. An occasional segmentation difficulty is the inclusion of part of the eyes in the extracted brain, due to similar MR signals in the brain and the optic tracts. It can always be solved by one or two simple manual threshold adjustments. Similar difficulties have been reported by Zijdenbos et al. [1994], who suggested that mathematical morphology might solve the problem. Contrary to previous morphological methods [Mangin et al., 1994; Alpert et al., 1996], our isotropic morphological operations are largely insensitive to voxel geometries. Consequently, modifications of the erosion and dilation parameters were only necessary when data sets were highly blurred by patient motion, while Alpert et al. [1996] reported modifications related to slice thickness. Our method is indifferent to even gross structural abnormalities (Fig. 1), because it only defines the brain as a central large mid-gray object mostly surrounded by dark or light layers. The 3D approach allows extraction of more than one 2D structure in some slices. The cerebellum and temporal lobes are thus correctly extracted, although they are three unconnected 2D objects in the lower axial slices. Our simulations and 
TABLE III. Registration accuracy with MIBI SPECT images and MRI images from patients with brain tumors

\begin{tabular}{lccc}
\hline & \multicolumn{3}{c}{ Distances between landmarks in MRI and SPECT } \\
\cline { 2 - 4 } Patient & $\begin{array}{c}\text { Left eye } \\
(\mathrm{mm})\end{array}$ & $\begin{array}{c}\text { Right eye } \\
(\mathrm{mm})\end{array}$ & $\begin{array}{c}\text { Choroid plexus } \\
\text { vs. ventricles }(\mathrm{mm})\end{array}$ \\
\hline 1 & 2.97 & 3.59 & 2.70 \\
2 & 8.74 & 5.29 & 1.05 \\
3 & 1.96 & 3.54 & 2.27 \\
4 & 6.45 & 4.76 & 5.17 \\
5 & 4.36 & 6.23 & 3.47 \\
6 & 7.09 & 5.93 & 9.24 \\
Average & 5.26 & 4.89 & 3.98 \\
\hline
\end{tabular}

clinical results suggest that this method was also appropriate for SPECT data sets despite their low spatial resolution.

Our matching method addresses the two major problems related to the registration of structural and functional datasets. First, brain surfaces extracted from functional and structural modalities do not represent the same physical reality and exhibit anatomicalfunctional shape differences (Fig. 5). We addressed this problem during surface matching by allowing the elimination of outliers on the SPECT surface. It proved efficient in our simulations, and useful in most clinical cases since even normal subjects do not show a uniformly perfused cortical rim because of the presense of nonperfused sulci (see the HMPAO SPECT image in Fig. 4). Our original generalized distance definition solves the second most important problem associated with multimodality registration, which is the difference of scanning range between modalities. With our distance definition, even registrations of partial 6.6-cm-thick (11 6-mm slices; Fig. 5) sagittal MRIs and partial axial SPECTs are routinely performed in a fully automatic manner. The longitudinal fissure yields a very specific surface shape which correctly guides the matching algorithm. The use of a new and efficient anisotrophic chamfer distance transform eliminates the need to reslice the image sets into cubic voxels, the associated loss of information, and the dramatic increase in computation time.

The provided supervision tools (real-time 3D and 2D displays, and 2D sections of the distance hypersurface) proved efficient for the quick detection and elimination of local minima of the distance function. With our corresponding distance 2D section, very large initial angles are immediately visible and can be easily corrected before launching Powell's algorithm. This eliminates the usually tedious and nonintuitive interac-
TABLE IV. Registration accuracy for HMPAO SPECT and MRI, using objective anatomical landmarks

\begin{tabular}{lcccc}
\hline \multicolumn{5}{c}{ Distances between landmarks in } \\
& \multicolumn{4}{c}{ MRI and SPECT } \\
\cline { 2 - 5 } & $\begin{array}{c}\text { Right eye } \\
(\mathrm{mm})\end{array}$ & $\begin{array}{c}\text { Sinus } \\
(\mathrm{mm})\end{array}$ & $\begin{array}{c}\text { Left eye } \\
(\mathrm{mm})\end{array}$ & $\begin{array}{c}\text { Centroid } \\
\text { triangle }(\mathrm{mm})\end{array}$ \\
\hline Normal controls & & & \\
Minimum & 0.94 & 0.94 & 0.94 & 0.70 \\
Maximum & 3.87 & 4.69 & 4.69 & 3.80 \\
Average & 2.27 & 2.99 & 2.65 & 1.49 \\
SD & 0.96 & 1.07 & 1.12 & 0.93 \\
Fronto-temporal dementia (FTD) & & \\
Minimum & 2.10 & 0.94 & 1.33 & 1.56 \\
Maximum & 10.69 & 10.69 & 9.65 & 6.16 \\
Average & 6.11 & 4.84 & 4.34 & 2.63 \\
SD & 3.34 & 2.64 & 2.66 & 1.26 \\
\hline
\end{tabular}

tion needed to find correct numerical parameter values for the initial positioning of partial brain scans. However, like more classical supervision methods such as multiple starting points or simulated annealing, our method gives no assurance of convergence towards the global minimum in a finite amount of time. It is important to note that this possible problem is not so much related to how well the algorithms are tuned as to the variability of the data sets, and the consequent variability in the shape of the generalized distance hypersurface. Our supervision scheme, however, proved efficient in all registrations performed. Our matching protocol proved automatic, reproducible, and objective in most cases. In difficult cases involving data sets of poor technical quality, however, expert subjectivity and manual adjustments are preferable to a failure.

Our simulations were useful for evaluating the algorithmic accuracy of our registration paradigm, and our highly abnormal clinical populations demonstrated the robustness of the method for routine use with a realistic variety of data sets. "Residual" distances (i.e., generalized distances between two surfaces after surface matching) were not used for validation because they do not accurately reflect the quality of a registration process [Hemler et al., 1995]. Our clinical results suggest that the spatial discrimination capability of SPECT with the Shimadzu camera was better than $5 \mathrm{~mm}$ when drawing regions on registered MRI. This combined the accuracy of the registration method and of the drawing of regions of interest on SPECT. The accuracy of the registration alone, as evaluated by our simulations, was typically around 1-2 $\mathrm{mm}$ (around 3-4 mm in Z-translation). The simula- 
tion results were satisying when considering that only 11 slices extracted from a data set with $12.4 \times 12.4 \times 20$ $\mathrm{mm}^{3}$ resolutions were used for mobile surface extraction, and registered to an MRI with 5-mm slice thickness. The method proved adequately insensitive to the addition of noise (the noise added in our simulations yielded images apparently much more degraded than real SPECT) and to the introduction of a large anatomical-functional brain shape difference. In this respect, it would be interesting to compare our method to that of Friston et al. [1995], using our technically imperfect, low-resolution data sets. Despite the accurate results obtained with our simulations, we do not recommend drawing regions of interest smaller than at least twice the imaging resolution. Also, the high resolution of MRI should not camouflage the low resolution of the original SPECT when interpreting quantitative localized measures. Finally, Strother et al. [1994] argued that multimodality registration methods based on comparisons of image intensities would be more accurate than surface-based methods. However, they only presented registrations of MRI with MRI, MRI with a displaced simulated PET obtained from the same MRI without introducing any focal anatomical-functional dissimilarity, and simulated PET with a displaced version of itself. Our results, however, suggest that our new surface-based method is capable of performing accurate anatomical-functional registrations with real data sets, in which structure and function may be significantly uncorrelated (see bottom row of Fig. 4).

\section{CONCLUSIONS}

Our method proved robust for mono- and multimodality registration, and addresses some of the prohibitive problems classically associated with registrations of highly variable populations and imaging techniques. These results were obtained by using several new and powerful image-processing techniques, including truly isotropic 3D morphological operations, dedicated contour interpolations, dedicated generalized distance calculation, anisotropic distance maps, and new supervision methods. Virtually any pair of 3D images could be registered, despite gross pathological or technical abnormalities. User interactions were sometimes required for images of poor quality, but these corrections were made quickly and easily. No extensive interaction, such as manual (or semiautomated) drawing of brain contours, manual outlining and pairing of landmarks, or manual editing of numerical registration parameter values, was required. Our method enables a wide range of neuroimaging correlation studies by allowing the combination of structure
(MRI), perfusion $\left({ }^{133} \mathrm{Xe}\right.$-calibrated HMPAO SPECT, and pMRI), focal activation (fMRI, and HMPAO SPECT), focal functional defects $\left({ }^{99} \mathrm{TC} \mathrm{MIBI},{ }^{201} \mathrm{TI}\right.$ and HMPAO SPECT, and gadolinium MRI), and brain chemistry (MRS). Correlation of these studies will enable a better understanding of how all these properties relate and interact in the normal and pathologic human brain.

\section{ACKNOWLEDGMENTS}

The authors thank Drs. I. Mena and B. Miller for their immensely valuable support during the intial phase of this project.

\section{REFERENCES}

Alpert NM, Bradshaw JF, Kennedy D, Correia JA (1990): The principal axes transformations-A method for image registration. J Nucl Med 31:1717-1722.

Alpert NM, Berdichevsky D, Levin Z, Morris ED, Fishman AJ (1996): Improved methods for image registration. Neuroimage 3:10-18.

Arata LK, Dhawan AP (1992): Iterative principal axes registration: A new algorithm for retrospective correlation of MRI-PET brain images. Proc 14th IEEE Eng Med Biol Soc (EMBS), Paris, pp 2776-2778.

Ardekani BA, Braun M, Kanno I, Hutton BF (1994): Automatic detection of intradural spaces in MR images. J Comput Assist Tomogr 18:963-969.

Barrow HG, Tenenbaum JM, Bolles RC, Wolf HC (1977): Parametric correspondance and the chamfer matching: Two new techniques for image matching. Proc 5th Int Joint Conf Artif Intell, Cambridge, pp 659-663.

Besl PJ, McKay ND (1992): A method for the registration of 3-D shapes. IEEE Trans Pat Anal Mach Intell 14:239-256.

Borgefors G (1986): Distance transformations in digital images. Comput Vision Graph Image Proc 34:344-371.

Bottomley PA (1987): Spatial localization in MRS spectroscopy in vivo. Ann NY Acad Sci 508:333-348.

Chang L, Yener GG, Miller BL, Mehringer M (1994): Magnetic resonance spectroscopy and single photon emission computed tomography in Alzheimer's disease: New directions. Facts Res Gerontol 7:295-307.

Chiu ML, Kronauge JF, Piwnica-Worms D (1990): Effect of mitochondrial and plasma membrane potentials on accumulation of hexakis (2-methoxyisobutylisonitrile) technetium(I) in cultured mouse fibroblasts. J Nucl Med 31:1646-1653.

Crosson B, Williamson DJG, Shukla SS, Honeyman JC, Nadeau SE (1994): A technique to localize activation in the human brain with technetium-99m-HMPAO SPECT: A validation study using visual stimulation. J Nucl Med 35:755-763.

DeCarli C, Maisog J, Murphy DGM, Teichberg D, Rapoport SI, Horwitz B (1992): Method for quantification of brain, ventricular, and subarachnoid CSF volumes for MR images. J Comput Assit Tomogr 16:274-284.

Dhawan AP, Arata LK, Levy AV, Mantil J. Interactive Principal Axes Registration method for analysis of MR-PET brain images. IEEE Transactions on Biomedical Engineering, 1995 Nov, 42(11):1079_ 87.

Ende G, Treuer H, Boesecke R (1991): Optimization and evaluation of landmark-based image correlation. Phys Med Biol 37:261-271. 
Ernst T, Kreis R, Ross BD (1993): Absolute quantitation of water metabolities in the human brain. I: Compartments and water. J Magn Reson 102:1-8.

Evans AC, Marret S, Torrescorzo J, Ku S, Collins L (1991): MRI-PET correlation in three dimensions using a volume-of-interest (voi) atlas. J Cereb Blood Flow Metab 11:69-78.

Fright WR, Linney AD (1993): Registration of 3-D head surfaces using multiple landmarks. IEEE Trans Med Imaging 12:515-520.

Friston KJ, Ashburner J, Frith CD, Poline JB, Heather JD, Frackowiak RSJ (1995): Spatial registration and normalization of images. Hum Brain Mapping 2:165-189.

Grabowski TJ, Damasio H, Frank R, Hichwa RD, Boles Ponto LL, Watkins GL (1995): A new technique for PET slice orientation and MRI-PET coregistration. Hum Brain Mapping 2:123-133.

Hasso AN (1993): Current status of enhanced magnetic resonance imaging in neurology. Invest Radiol 28:3-20.

Hemler PF, Napel S, Sumanaweera TS, Pichumani R, van den Elsen PA, Martin D, Drace J, Adler JR, Perkash I (1995): Registration error quantification of a surface-based multimodality image fusion system. Med Phys 22:1049-1056.

Holman BL, Zimmerman RE, Johnson KA, Carvalho PA, Schwartz RB, Loeffler JS, Alexander E, Pelizzari CA, Chen GTY (1991): Computer-assisted superimposition of magnetic resonance and high-resolution technetium-99-m-HMPAO and thallium-201 SPECT images of the brain. J Nucl Med 32:1478-1484.

Jiang H, Robb RA, Holton KSA (1992): A new approach to 3-D registration of multimodality medical images by surface matching. SPIE Vis Biomed Comput 1808:196-213.

Kanno I, Lassen NA (1979): Two methods for calculating regional cerebral blood flow from emission computed tomography of inert gas concentration. J Comput Assist Tomogr 5:216-224.

Kreis R, Ernst T, Ross BD (1993): Absolute quantitation of water metabolites in the human brain. II: Metabolite concentrations. J Magn Reson 102:9-19.

Levin DN, Pelizzari CA, Chen GTY, Chen CT, Cooper MD (1988): Retrospective geometric correlation of $\mathrm{MR}, \mathrm{CT}$, and PET images. Radiology 169:817-823.

Loats H (1993): CT and SPECT image registration and fusion for spatial localization of metastatic processes using radiolabeled monoclonals. J Nucl Med 34:562-566.

Malandain G (1992): Filtrage, topologie et mise en correspondance d'images médicales multidimensionnelles. Thèse de Doctorat, Ecole Centrale de Paris.

Mangin JF, Frouin V, Bloch I, Bendriem B, Lopez-Krahe J (1994): Fast nonsupervised 3D registration of PET and MRI images of the brain. J Cereb Blood Flow Metab 14:749-762.
Masdeu JC, Brass LM, Holman BL, Kushner MJ (1994): Brain single-photon emission computed tomography. Neurology 44: 1970-1977.

Moshfeghi M, Rusinek H (1992): Three-dimensional registration of multimodality medical images using the principal axes technique. Philips J Res 47:81-97.

Pelizzari CA, Chen GTY, Spelbring DR,Weichselbaum RR, Chen CT (1989): Accurate three-dimensional registration of CT, PET, and/or MR images of the brain. J Comput Assist Tomogr 13:20-26.

Powell MJD (1964): An efficient method for finding the minimum of a function of several variables without calculating derivatives. Comput J 7:155-163.

Press WH, Teukolsky SA, Vetterling WT, Flannery BP (1992): Numerical Recipes in C. Cambridge, UK: Cambridge University Press

Schmitt M, Mattioli J (1994): Morphologie Mathématique. Paris: Masson.

Strother SC, Anderson JR, Xu XL, Liow JS, Bonar DC, Rottenberg DA (1994): Quantitative comparisons of image registration techniques based on high-resolution MRI of the brain. J Comput Assist Tomogr 18:954-962.

Turkington TG, Hoffman JM, Jaszczak RJ, MacFall JR, Harris CC, Kilts CD, Pelizzari CA, Coleman RE (1995): Accuracy of surface fit registration for PET and MR brain images using full and incomplete brain surfaces. J Comput Assist Tomogr 19:117-124.

Van den Elsen PA, Pol ED, Viergever MA (1993): Medical image matching-A review with classification. IEEE Eng Med Biol 12:26-39.

Van Herk M, Kooy HM (1994): Automatic three-dimensional correlation of CT-CT, CT-MRI and CT-SPECT using chamfer matching. Med Phys 21:1163-1177.

Wacken PFM (1994): Chamfer metrics in mathematical morphology. J Math Imaging Vision 4:233-253.

Wang C, Pahl JJ, Hogue RE (1994): A method for co-registering three-dimensional multimodality brain images. Comput Methods Programs Biomed 44:131-140.

Woods RP, Mazziotta JC, Cherry SR (1993): MRI-PET registration with automated algorithm. J Comput Assist Tomogr 17:536-546.

Yioshizawa T, Satoh M, Matsumara A, Hyodo A, Enomoto T, Ishikawa H, Pathak PN, Nose T (1995): Functional brain mapping employing modeling and statistical procedures to detect focal changes of CBF via SPECT. J Cereb Blood Flow Metab 15:121.

Zijdenbos AP, Dawant BM, Margolin RA (1994): Automatic detection of intracranial contours in MR images. Comput Med Imaging Graph 18:11-23. 